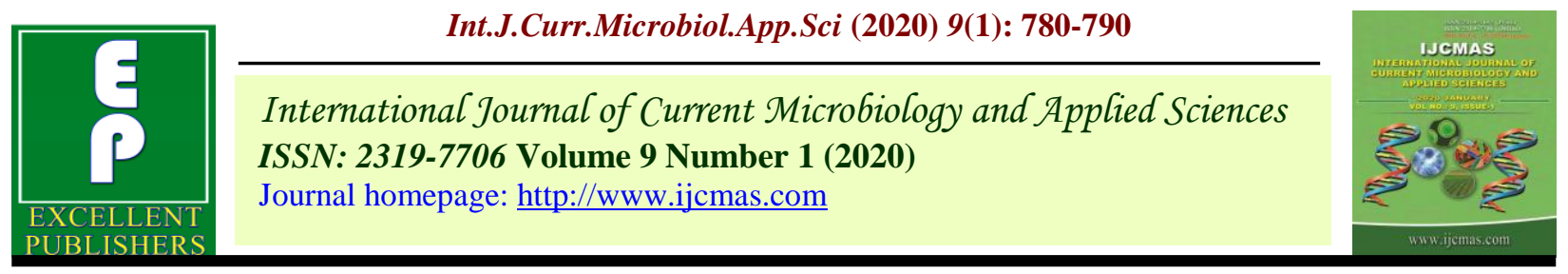

Original Research Article

https://doi.org/10.20546/ijcmas.2020.901.084

\title{
Morphological Characterization and Evaluation of Mungbean [Vigna radiate (L.) Wilczek] Germplasm for Various Yield Attributing Traits
}

\author{
Sarfraz Ahmad* and Vikas Belwal
}

Department of Genetics and Plant Breeding, G.B. Pant University of Agriculture and
Technology, Pantnagar, Uttarkhand-263 145, India

*Corresponding author

\begin{tabular}{|l|}
\hline Ke y w o r d s \\
Green gram, \\
Morphological \\
characterization, \\
Mungbean, Vigna \\
radiata
\end{tabular}

\section{A B S T R A C T}

The present investigation was carried out with 112 diverse genotypes along with 5 checks of mungbean [Vigna radiata (L.) Wilczek] during Kharif season. Data were recorded on various morphological and agronomic characters including hypocotyl pigmentation, days to 50 percent flowering, plant height, number of leaves, number of branches, number of clusters, number of pods per cluster, total number of pods per plant, pod length, pod diameter, pod wall thickness, seed length, seed diameter, 100-seed weight, seed density and seed yield. Analysis of variance revealed significant differences among all the genotypes for all the characters studied. Wide range of variation had been observed for all the characters except hypocotyl pigmentation. Eight genotype were at par with respect to seed yield per plot from the best performing check PM-6 $(0.55 \mathrm{~kg})$, these genotype were as PM 06-46 (0.71 kg), MH-318 (0.69 kg), VC 1997 A (0.68 kg), SML-668 (0.67 kg), COGG-912 (0.62 kg), Barimung-5 (0.61 kg), PM 03-4 (0.59 kg) and TM 96-2 $(0.58 \mathrm{~kg})$. The study also provides information about the performance of some of the promising genotypes in respect of morphological and agronomic characters and identifies some prominent genotypes. These genoypes may be further used for parental selection in breeding programmes for improvement of yield and various qualitative and quantitative characteristics in mungbean.

\section{Introduction}

Mungbean [Vigna radiata (L.) Wilczek] is one of the important grain legumes of global economic importance. In India, it is the third most important pulse crop after chickpea and pigeon pea. It belongs to family Fabaceae (syn. Leguminoseae) and sub family 
Papilionaceae. It is believed to have been domesticated from Vigna radiata var. sublobata. Mungbean, also known as green gram, has originated in Indian sub-continent. Mungbeans are mainly cultivated in India, China, Thailand, Philippines, Indonesia, Burma, and Bangladesh and in hot and dry regions of South Europe and Southern USA (Singh et al., 2005). It is priced among pulse crops as its seeds are high in essential dietary protein, easily digested and produce low flatulence when consumed as food (Lakhanpaul et al., 2000). Mungbean is primarily used as dhal. Green pods are used as vegetables and is an excellent source of easily digestible protein (22-25\%) (Rao et al., 1964). Easy assimilability, short duration of cropping and the ease with which it could be grown as a mixture with other crops makes it superior than other legumes.

In any crop breeding programme, germplasm evaluation plays a vital role in identification of superior genotypes for different qualitative and quantitative characters which may be further used to create variability by hybridization. Plant genetic resources are the most valuable and essential basic raw materials to meet the current and future needs of crop improvement programmes (Paroda et al., 1991). The genotypes which are suitable at present may not be suited in future due to change in environment and susceptibility to biotic and abiotic stresses. The evaluation of germplasm to identify genotypes capable of withstanding/ adapting to the newer stresses arising from the changing environment is, therefore, a continuous and indispensable process in crop improvement. The present investigation was, therefore, undertaken to morphological characterization and evaluation of mungbean germplasm for yield and attributing characters and identifies superior genotypes among them for various characters under studied.

\section{Materials and Methods}

The experiment on morphological characterization and evaluation of Mungbean (Vigna radiata) germplasm for various yield attributing traits were conducted at N. E. Borlaug Crop Research Center of G.B. Pant University of Agriculture and Technology, Pantnagar, India, during Kharif 2010-2011. The experimental material for the present study consisted of 112 germplasm lines of Vigna radiata along with 5 checks viz. Pant Mung-2 (PM-2), Pant Mung-3 (PM-3), Pant Mung-4 (PM-4), Pant Mung-5 (PM-5) and Pant Mung-6 (PM-6). The germplasm strains selected for the investigation were genetically diverse and exhibited wide range of variation for the qualitative and quantitative characters (Table 2). The field experiment was laid out in Augmented Block Design (Federer, 1961), which consisted 4 blocks with 5 checks repeated in each block. Two rows of each entry were planted with keeping row length 4 meter. Row to row distance was kept $30 \mathrm{~cm}$ and plant to plant distance was maintained at $10 \mathrm{~cm}$.

\section{Observations recorded}

Total 16 characters were recorded following DUS guideline of mungbean, these were as hypocotyl pigmentation (red or green), days to 50 percent flowering, plant height $(\mathrm{cm})$, number of leaves, number of branches, number of clusters, number of pods per cluster, total number of pods per plant, pod length $(\mathrm{cm})$, pod diameter $(\mathrm{mm})$, pod wall thickness $(\mathrm{mm})$, seed length $(\mathrm{mm})$, seed diameter $(\mathrm{mm}), 100$-seed weight $(\mathrm{gm})$, seed density $(\mathrm{gm} / \mathrm{ml})$ and seed yield per plot (kg/plot). Observations were recorded on the whole plot basis for the characters hypocotyl pigmentation and days to 50 percent flowering, whereas the characters like plant height, number of branches, number of clusters, number of pods per cluster, total 
number of pods per plant and number of leaves were taken on five randomly selected competitive plants from each line. The average values for these plants were calculated and used for the statistical analysis. Pod length, pod diameter and pod wall thickness were recorded on 10 randomly selected pods in each entry. Seed length and seed diameter were recorded on 10 randomly selected seeds in each entry, while 100-seed weight and seed density recorded on three sets (replications) of 100 randomly selected seeds in each entry.

Statistical analysis: The data were analyzed using IRRISTAT software.

\section{Results and Discussion}

The analysis of variance for the characters days to 50 percent flowering, plant height, number of leaves, number of branches, number of clusters, number of pods per cluster, total number of pods per plant, pod length, pod diameter, pod wall thickness, seed length, seed diameter, 100-seed weight, seed density and seed yield per plot was performed in augmented block design (ABD) and the results along with checks mean, Coefficient of variation and least significant differences for different characters are presented in table 1.

The statistical analysis revealed highly significant differences among the genotypes for all the characters studied which clearly indicated that there was sufficient variability for each character among the genotypes selected for the study. The adjusted mean value for all the characters of different genotypes, general mean and range of variation are presented in table 2 . The list of top five superior genotypes for different characters is presented in table 3 .

Out of 112 genotypes 100 had red pigmentation on the hypocotyl while 12 genotypes had green pigmentation. These 12 genotypes were Barimung-5, UPM 03-18, PM 08-16, PM 08-2, 45-8-1, NDM 5-3, VC 6040 A, ML 133, Pusa Ratna, PM 06-39, PM 06-43 and KM 09-174. The range of variation in days to 50 percent flowering varied from 31 days (CN 9-5) to 48 days (PM 06-34), plant height varied from $34.17 \mathrm{~cm}$ (IPM 02-19) to $84.49 \mathrm{~cm}$ (PM 06-57), number of branches lied between 2.35 (VC 7960-88) to 9.25 (PU 03-11). Number of clusters ranged from 3.83 (PM 06-43) to 38.03 (PM 06-32) and number of pods per cluster ranged between 3.17 (SML 668) to 5.67 (PM 06-46). Total number of pods per plant ranged from 18.10 (Harsha) to 88.14 (PM 06-46) and number of leaves ranged from 11.18 (NH-54) 61.36 (PM 0632).

Average pod length varied from $4.10 \mathrm{~cm}$ (Vigna radiata var sublobata) to $10.32 \mathrm{~cm}$ (NH-54), average pod diameter was between $2 \mathrm{~mm}$ (Vigna radiata var sublobata) to 6.01 mm (VC 1997 A) and average pod wall thickness varied from $0.14 \mathrm{~mm}$ (RMG 991 and VC7960) to $0.28 \mathrm{~mm}$ (Barimung-5). Average seed length ranged from $2.63 \mathrm{~mm}$ (Vigna radiata var sublobata) to $5.55 \mathrm{~mm}$ (VC 6790A) and average seed diameter was between $1.73 \mathrm{~mm}$ (Vigna radiata var sublobata) to $4.02 \mathrm{~mm}$ (VC $1997 \mathrm{~A}$ ).

Range of variation for 100-seed weight varied from $2.20 \mathrm{~g}$ (Vigna radiata var sublobata) to $5.85 \mathrm{~g}$ (VC $1997 \mathrm{~A}$ ) and density of seed ranged from $1.12 \mathrm{~g} / \mathrm{ml}$ (PM 08-1) to $1.72 \mathrm{~g} / \mathrm{ml}$ (VC 1997 A). Seed yield per plot varied from $0.04 \mathrm{~kg}$ (Vigna radiata var sublobata) to 0.71 $\mathrm{kg}$ (PM 06-46). However, total eight genotype were at par with respect to seed yield per plot from the best performing check PM-6 (0.55 $\mathrm{kg})$, these genotype were as PM 06-46 (0.71 $\mathrm{kg}), \mathrm{MH}-318(0.69 \mathrm{~kg}), \mathrm{VC} 1997 \mathrm{~A}(0.68 \mathrm{~kg})$, SML-668 (0.67 kg), COGG-912 (0.62 kg), Barimung-5 (0.61 kg), PM 03-4 (0.59 kg) and TM 96-2 (0.58 kg). 
Table.1 Analysis of Variance in ABD for different characters of mungbean genotypes

\begin{tabular}{|c|c|c|c|c|c|c|c|c|c|c|c|c|c|c|}
\hline \multirow[t]{2}{*}{ S. No. } & \multirow[t]{2}{*}{ Characters } & \multicolumn{5}{|c|}{ Mean value of Checks } & \multicolumn{3}{|c|}{ Mean Sum of Squares } & \multirow[t]{2}{*}{$\mathbf{C V}$} & \multirow[t]{2}{*}{$\mathbf{C M}$} & \multirow[t]{2}{*}{ AVSB } & \multirow[t]{2}{*}{ AVDB } & \multirow[t]{2}{*}{ AVAC } \\
\hline & & $\begin{array}{l}\text { PM- } \\
2\end{array}$ & $\begin{array}{c}\text { PM- } \\
\mathbf{3}\end{array}$ & $\begin{array}{l}\text { PM- } \\
4\end{array}$ & $\begin{array}{l}\text { PM- } \\
5\end{array}$ & PM-6 & Blocks & Checks & Error & & & & & \\
\hline 1. & Days to $50 \%$ flowering & 39.50 & 39.00 & 39.50 & 36.75 & 42.25 & 5.60 & 15.33 & 6.06 & 5.15 & 3.79 & 7.58 & 8.31 & 6.57 \\
\hline 2. & Plant height (cm) & 53.55 & 68.50 & 58.30 & 55.40 & 75.30 & 2.71 & $347.57 * *$ & 15.31 & 5.17 & 6.03 & 12.00 & 13.20 & 10.44 \\
\hline 3. & No. of leaves & 30.35 & 33.15 & 22.33 & 24.65 & 41.60 & 0.99 & $231.27 * *$ & 4.90 & 6.92 & 3.41 & 6.82 & 7.47 & 5.91 \\
\hline 4. & No. of branches & 3.30 & 4.50 & 3.60 & 3.67 & 4.67 & 0.07 & $1.45 * *$ & 0.04 & 4.96 & 0.30 & 0.61 & 0.67 & 0.53 \\
\hline 5. & No. of clusters & 14.75 & 15.51 & 20.00 & 17.20 & 23.50 & 2.43 & $51.45 * *$ & 1.87 & 6.95 & 2.10 & 4.21 & 4.61 & 3.65 \\
\hline 6. & No. of pods/cluster & 3.95 & 4.15 & 4.20 & 3.90 & 4.33 & 0.09 & 0.13 & 0.05 & 5.51 & 0.36 & 0.72 & 0.79 & 0.63 \\
\hline 7. & Total no. of pods/plant & 55.15 & 53.27 & 39.20 & 29.20 & 66.65 & 6.53 & $855.24 * *$ & 15.91 & 7.96 & 6.14 & 12.29 & 13.46 & 10.64 \\
\hline 8. & Pod length $(\mathrm{cm})$ & 7.08 & 7.11 & 8.08 & 8.37 & 6.86 & 0.59 & $1.82 * *$ & 0.17 & 4.43 & 0.64 & 1.29 & 1.41 & 1.12 \\
\hline 9. & Pod diameter (mm) & 4.28 & 4.44 & 4.60 & 4.96 & 4.53 & 0.03 & $0.25^{* *}$ & 0.03 & 2.88 & 0.28 & 0.57 & 0.62 & 0.49 \\
\hline 10. & $\begin{array}{l}\text { Pod wall thickness } \\
\text { (mm) }\end{array}$ & 0.16 & 0.18 & 0.20 & 0.20 & 0.20 & 0.00003 & $0.001 * *$ & 0.00001 & 1.26 & 0.01 & .01 & 0.01 & 0.01 \\
\hline 11. & Seed length (mm) & 4.13 & 3.77 & 3.86 & 4.07 & 3.75 & 0.01 & $0.12 * *$ & 0.01 & 2.24 & 0.16 & 0.31 & 0.34 & 0.27 \\
\hline 12. & Seed diameter $(\mathrm{mm})$ & 3.13 & 3.12 & 3.10 & 3.62 & 3.05 & 0.00079 & $0.22 * *$ & 0.0064 & 1.87 & 0.12 & 0.25 & 0.27 & 0.21 \\
\hline 13. & 100-seed weight (gm) & 3.23 & 3.17 & 3.46 & 4.82 & 3.26 & 0.04 & $1.41 * *$ & 0.16 & 4.79 & 0.14 & 0.28 & 0.31 & 0.24 \\
\hline 14. & Seed density (gm/ml) & 1.27 & 1.35 & 1.35 & 1.43 & 1.34 & 0.002 & 0.032 & 0.07 & 3.53 & 0.04 & 0.08 & 0.09 & 0.07 \\
\hline 15. & Seed yield per plot $(\mathbf{k g})$ & 0.45 & 0.46 & 0.44 & 0.52 & 0.55 & 0.002 & $0.038 * *$ & 0.022 & 8.85 & 0.07 & 0.02 & 0.03 & 0.02 \\
\hline
\end{tabular}

* Significant at $5 \%$ of level of probability

** Significant at $1 \%$ of level of probability

$\mathrm{CM}=$ least significant difference between the means of two check varieties,

AVSB = least significant difference between adjusted values of two selections in the same block,

AVDB = least significant difference between adjusted value of two selection in different blocks,

$\mathrm{AVAC}=$ least significant difference between an adjusted selection value and a check mean 
Table.2 Adjusted mean for various characters of mungbean genotypes

\begin{tabular}{|c|c|c|c|c|c|c|c|c|c|c|c|c|c|c|c|c|}
\hline $\begin{array}{l}\text { S. } \\
\text { N. }\end{array}$ & Genotypes & $\begin{array}{c}\text { Days to } \\
50 \% \\
\text { flowering }\end{array}$ & $\begin{array}{c}\text { Plant } \\
\text { height } \\
(\mathrm{cm})\end{array}$ & $\begin{array}{l}\text { No. of } \\
\text { leaves }\end{array}$ & $\begin{array}{c}\text { No. of } \\
\text { branches }\end{array}$ & $\begin{array}{l}\text { No. of } \\
\text { cluster }\end{array}$ & $\begin{array}{l}\text { No. of } \\
\text { pods/ } \\
\text { cluster }\end{array}$ & $\begin{array}{l}\text { Total } \\
\text { no. of } \\
\text { pods/ } \\
\text { plant }\end{array}$ & $\begin{array}{c}\text { Pod } \\
\text { length } \\
(\mathrm{cm})\end{array}$ & $\begin{array}{l}\text { Pod } \\
\text { dia. } \\
(\mathbf{m m})\end{array}$ & $\begin{array}{c}\text { Pod wall } \\
\text { thickness } \\
(\mathbf{m m})\end{array}$ & $\begin{array}{c}\text { Seed } \\
\text { length } \\
(\mathbf{m m})\end{array}$ & $\begin{array}{c}\text { Seed } \\
\text { dia. } \\
(\mathrm{mm})\end{array}$ & $\begin{array}{c}100- \\
\text { seed } \\
\text { wt } \\
(g)\end{array}$ & $\begin{array}{c}\text { Seed } \\
\text { density } \\
(\mathrm{g} / \mathrm{ml})\end{array}$ & $\begin{array}{c}\text { Seed } \\
\text { yield / } \\
\text { plot }(\mathbf{k g})\end{array}$ \\
\hline 1 & PM-03-2 & 39 & 41.25 & 24.30 & 9.05 & 9.55 & 4.59 & 31.38 & 9.03 & 5.19 & 0.22 & 4.68 & 3.47 & 4.45 & 1.37 & 0.35 \\
\hline 2 & PM 03-4 & 39 & 54.45 & 23.30 & 5.05 & 15.55 & 4.39 & 50.98 & 8.27 & 5.22 & 0.22 & 4.92 & 3.70 & 4.58 & 1.37 & 0.59 \\
\hline 3 & PM 03-5 & 37 & 56.85 & 28.70 & 8.05 & 14.55 & 3.59 & 40.98 & 8.18 & 4.97 & 0.19 & 4.19 & 3.17 & 4.02 & 1.34 & 0.35 \\
\hline 4 & PM 03-7 & 37 & 60.45 & 23.30 & 6.45 & 10.95 & 4.19 & 38.98 & 7.95 & 5.05 & 0.18 & 4.86 & 3.48 & 4.50 & 1.38 & 0.39 \\
\hline 5 & PM 03-9 & 36 & 67.85 & 30.90 & 9.05 & 9.95 & 4.19 & 46.58 & 7.50 & 4.77 & 0.18 & 3.99 & 3.51 & 3.89 & 1.36 & 0.34 \\
\hline 6 & PM 03-11 & 41 & 77.25 & 32.70 & 9.25 & 10.15 & 4.39 & 50.38 & 7.87 & 4.92 & 0.19 & 4.76 & 3.57 & 4.37 & 1.38 & 0.49 \\
\hline 7 & PM 03-12 & 43 & 61.25 & 36.30 & 6.85 & 7.75 & 4.19 & 42.38 & 7.00 & 4.53 & 0.16 & 4.38 & 3.36 & 3.63 & 1.40 & 0.24 \\
\hline 8 & PM 03-13 & 39 & 69.65 & 28.50 & 6.65 & 9.55 & 4.19 & 34.38 & 8.37 & 4.94 & 0.21 & 4.14 & 3.12 & 4.16 & 1.37 & 0.31 \\
\hline 9 & PM 03-15 & 36 & 74.45 & 32.50 & 9.05 & 14.95 & 4.39 & 38.78 & 7.67 & 4.89 & 0.19 & 4.52 & 3.47 & 4.43 & 1.39 & 0.35 \\
\hline 10 & PM 03-16 & 36 & 54.85 & 25.10 & 5.65 & 7.35 & 4.59 & 28.58 & 6.66 & 4.58 & 0.17 & 4.02 & 3.20 & 3.68 & 1.41 & 0.13 \\
\hline 11 & PM 03-17 & 35 & 52.25 & 22.10 & 4.25 & 8.75 & 4.39 & 32.98 & 7.99 & 5.20 & 0.22 & 4.77 & 3.53 & 4.17 & 1.40 & 0.30 \\
\hline 12 & PM 03-18 & 39 & 59.85 & 21.10 & 3.65 & 8.55 & 4.39 & 32.78 & 7.66 & 4.72 & 0.20 & 4.47 & 3.16 & 3.88 & 1.39 & 0.22 \\
\hline 13 & PM 03-19 & 35 & 64.05 & 23.50 & 5.25 & 12.95 & 4.19 & 45.18 & 7.87 & 5.16 & 0.20 & 4.05 & 3.12 & 4.09 & 1.28 & 0.38 \\
\hline 14 & PM 03-20 & 35 & 55.25 & 22.30 & 3.65 & 9.75 & 3.99 & 30.98 & 9.25 & 5.40 & 0.24 & 4.92 & 3.50 & 4.76 & 1.30 & 0.38 \\
\hline 15 & PM 03-22 & 37 & 62.25 & 31.30 & 4.45 & 15.75 & 4.19 & 44.98 & 8.37 & 4.97 & 0.23 & 4.68 & 3.43 & 4.29 & 1.38 & 0.46 \\
\hline 16 & PM 03-23 & 36 & 60.05 & 20.30 & 3.25 & 10.95 & 3.19 & 29.18 & 8.91 & 5.20 & 0.21 & 5.18 & 3.58 & 4.95 & 1.37 & 0.36 \\
\hline 17 & PM 03-24 & 41 & 55.45 & 28.10 & 3.85 & 12.35 & 3.59 & 36.78 & 7.76 & 4.79 & 0.19 & 4.18 & 3.29 & 4.04 & 1.36 & 0.28 \\
\hline 18 & PM 03-25 & 39 & 56.05 & 31.90 & 3.65 & 12.35 & 4.39 & 46.18 & 8.41 & 4.86 & 0.25 & 4.54 & 3.43 & 4.56 & 1.40 & 0.50 \\
\hline 19 & PM-3(M) & 40 & 71.05 & 34.90 & 3.65 & 15.55 & 4.19 & 51.58 & 7.04 & 4.39 & 0.19 & 4.25 & 3.40 & 3.03 & 1.18 & 0.19 \\
\hline 20 & PM 06-4 & 39 & 59.25 & 32.30 & 4.45 & 15.15 & 3.59 & 37.58 & 6.93 & 4.46 & 0.20 & 4.29 & 3.17 & 3.33 & 1.38 & 0.17 \\
\hline 21 & PM 06-16 & 42 & 64.25 & 22.50 & 3.45 & 19.75 & 3.99 & 53.38 & 7.30 & 4.49 & 0.19 & 4.14 & 3.18 & 3.01 & 1.30 & 0.24 \\
\hline 22 & PM 06-31 & 47 & 72.45 & 32.10 & 4.05 & 21.75 & 3.99 & 55.98 & 6.72 & 4.63 & 0.23 & 4.13 & 3.23 & 3.22 & 1.36 & 0.28 \\
\hline 23 & PM 06-41 & 41 & 70.05 & 25.10 & 4.45 & 24.75 & 3.79 & 43.58 & 6.54 & 4.86 & 0.21 & 4.34 & 3.39 & 3.56 & 1.40 & 0.25 \\
\hline 24 & PM 06-42 & 42 & 82.65 & 31.90 & 3.45 & 17.15 & 3.59 & 47.58 & 6.71 & 4.58 & 0.23 & 4.16 & 3.23 & 3.23 & 1.38 & 0.23 \\
\hline 25 & PM 06-45 & 41 & 74.85 & 16.70 & 3.45 & 15.15 & 4.19 & 36.58 & 7.72 & 4.54 & 0.22 & 3.72 & 3.09 & 3.30 & 1.27 & 0.17 \\
\hline 26 & PM 06-48 & 39 & 69.45 & 21.10 & 3.65 & 12.75 & 4.59 & 24.38 & 7.67 & 4.81 & 0.19 & 4.61 & 3.57 & 3.56 & 1.30 & 0.11 \\
\hline 27 & PM 06-49 & 39 & 70.85 & 35.90 & 3.45 & 18.55 & 3.79 & 49.58 & 7.16 & 4.93 & 0.20 & 4.87 & 3.33 & 3.48 & 1.32 & 0.30 \\
\hline
\end{tabular}




\begin{tabular}{|c|c|c|c|c|c|c|c|c|c|c|c|c|c|c|c|c|}
\hline 28 & PM 06-51 & 41 & 79.65 & 42.50 & 4.65 & 17.15 & 5.39 & 50.38 & 7.75 & 4.91 & 0.21 & 3.95 & 3.49 & 3.56 & 1.35 & 0.36 \\
\hline 29 & PM 06-57 & 42 & 84.49 & 43.58 & 3.55 & 16.59 & 4.39 & 53.80 & 7.50 & 4.47 & 0.22 & 4.20 & 3.35 & 3.40 & 1.38 & 0.33 \\
\hline 30 & MH-429 & 37 & 64.49 & 26.18 & 3.55 & 9.59 & 3.99 & 37.60 & 7.79 & 4.40 & 0.20 & 4.01 & 3.03 & 3.44 & 1.31 & 0.19 \\
\hline 31 & NH-54 & 34 & 59.49 & 11.18 & 3.35 & 12.79 & 3.59 & 29.80 & 10.32 & 5.24 & 0.20 & 5.03 & 3.49 & 3.28 & 1.30 & 0.25 \\
\hline 32 & NM-94 & 39 & 60.49 & 18.58 & 3.15 & 14.39 & 3.59 & 37.40 & 7.83 & 4.43 & 0.19 & 4.40 & 3.38 & 3.61 & 1.35 & 0.22 \\
\hline 33 & NM-1 (Mutant) & 39 & 47.69 & 27.18 & 2.55 & 9.79 & 3.39 & 24.40 & 7.20 & 4.39 & 0.19 & 3.84 & 3.43 & 3.69 & 1.42 & 0.10 \\
\hline 34 & ICM-1 & 37 & 55.49 & 27.58 & 3.15 & 9.39 & 4.39 & 34.00 & 7.20 & 4.40 & 0.19 & 3.84 & 3.43 & 3.07 & 1.31 & 0.12 \\
\hline 35 & VC $6790 \mathrm{~A}$ & 33 & 68.89 & 31.18 & 2.95 & 12.59 & 3.99 & 28.60 & 8.86 & 5.71 & 0.26 & 5.55 & 3.81 & 5.31 & 1.35 & 0.42 \\
\hline 36 & VC 6769 (57-99) & 32 & 57.69 & 21.78 & 3.55 & 13.99 & 3.99 & 42.20 & 7.86 & 4.87 & 0.20 & 3.83 & 3.30 & 3.75 & 1.29 & 0.29 \\
\hline 37 & VC 7960-88 & 33 & 52.29 & 20.18 & 2.35 & 10.79 & 3.59 & 27.20 & 8.25 & 5.32 & 0.22 & 4.73 & 3.53 & 4.38 & 1.49 & 0.29 \\
\hline 38 & BDYR-1 & 37 & 79.49 & 46.38 & 4.75 & 26.19 & 4.19 & 61.40 & 7.80 & 4.59 & 0.22 & 4.35 & 3.36 & 3.88 & 1.32 & 0.47 \\
\hline 39 & BDYR-2 & 37 & 64.69 & 31.98 & 4.15 & 18.99 & 3.79 & 45.20 & 8.08 & 4.83 & 0.24 & 4.04 & 3.18 & 3.91 & 1.39 & 0.38 \\
\hline 40 & Barimung-4 & 44 & 70.49 & 45.58 & 6.15 & 28.59 & 3.79 & 57.00 & 7.75 & 4.42 & 0.22 & 4.20 & 3.15 & 3.41 & 1.31 & 0.44 \\
\hline 41 & Barimung-5 & 37 & 53.69 & 29.78 & 3.55 & 12.59 & 4.39 & 34.00 & 8.36 & 5.90 & 0.28 & 4.28 & 3.81 & 5.52 & 1.53 & 0.61 \\
\hline 42 & Barimung-7 & 37 & 60.29 & 17.78 & 3.35 & 11.79 & 3.99 & 37.60 & 7.07 & 4.22 & 0.23 & 3.84 & 3.45 & 3.30 & 1.37 & 0.36 \\
\hline 43 & Barisal Local & 37 & 62.29 & 18.38 & 3.55 & 12.59 & 3.79 & 37.40 & 9.21 & 4.79 & 0.21 & 4.21 & 3.28 & 3.90 & 1.54 & 0.40 \\
\hline 44 & Mauritius Local & 32 & 67.09 & 40.78 & 5.35 & 25.19 & 4.39 & 62.00 & 7.65 & 4.43 & 0.18 & 3.81 & 3.35 & 3.84 & 1.48 & 0.50 \\
\hline 45 & Pusa Vishal & 32 & 57.69 & 31.58 & 3.55 & 16.19 & 3.79 & 41.40 & 7.95 & 4.88 & 0.21 & 4.42 & 3.53 & 4.00 & 1.15 & 0.27 \\
\hline 46 & UPM 02-16 & 39 & 56.09 & 22.98 & 2.75 & 12.59 & 3.39 & 36.20 & 9.42 & 4.80 & 0.23 & 4.48 & 3.27 & 3.77 & 1.34 & 0.32 \\
\hline 47 & UPM 98-1 & 31 & 56.49 & 31.38 & 3.95 & 18.39 & 3.59 & 38.80 & 8.02 & 4.66 & 0.20 & 4.17 & 3.46 & 3.71 & 1.39 & 0.28 \\
\hline 48 & UPM-98 & 39 & 50.09 & 30.38 & 2.75 & 10.79 & 3.59 & 32.40 & 9.08 & 5.09 & 0.23 & 5.00 & 3.82 & 4.53 & 1.42 & 0.38 \\
\hline 49 & UPM 03-18 & 39 & 60.09 & 28.78 & 3.15 & 10.19 & 3.59 & 38.40 & 7.56 & 4.53 & 0.21 & 4.35 & 3.49 & 4.27 & 1.39 & 0.29 \\
\hline 50 & UPM 98-10 & 42 & 71.69 & 38.58 & 4.15 & 19.79 & 4.19 & 49.40 & 8.00 & 4.49 & 0.26 & 3.92 & 3.16 & 3.32 & 1.26 & 0.27 \\
\hline 51 & UPM 93-3 & 42 & 70.69 & 34.58 & 4.75 & 25.19 & 3.99 & 56.80 & 8.06 & 4.76 & 0.22 & 4.09 & 3.29 & 3.66 & 1.31 & 0.42 \\
\hline 52 & UPM 99-3 & 39 & 50.69 & 28.98 & 3.55 & 15.39 & 3.79 & 44.20 & 7.40 & 4.58 & 0.17 & 4.11 & 3.37 & 3.75 & 1.32 & 0.27 \\
\hline 53 & PM 08-16 & 42 & 55.89 & 29.98 & 3.55 & 18.19 & 4.19 & 51.80 & 7.98 & 4.41 & 0.18 & 3.94 & 2.94 & 3.31 & 1.24 & 0.28 \\
\hline 54 & PM 08-2 & 37 & 48.69 & 22.58 & 2.54 & 13.19 & 4.39 & 45.00 & 7.69 & 4.41 & 0.17 & 3.96 & 3.20 & 2.86 & 1.21 & 0.17 \\
\hline 55 & PM 08-1 & 33 & 58.89 & 32.38 & 4.15 & 20.79 & 4.19 & 51.20 & 8.54 & 4.83 & 0.16 & 4.57 & 3.56 & 4.25 & 1.12 & 0.41 \\
\hline 56 & MH-318 & 37 & 48.69 & 34.78 & 4.15 & 17.79 & 3.99 & 57.40 & 9.46 & 4.91 & 0.24 & 4.46 & 3.73 & 5.24 & 1.56 & 0.69 \\
\hline 57 & $\begin{array}{l}\text { Mauritius } \\
\text { Mung-1 }\end{array}$ & 32 & 50.09 & 29.18 & 2.81 & 8.22 & 3.97 & 31.50 & 7.21 & 4.48 & 0.19 & 4.07 & 3.32 & 3.73 & 1.36 & 0.16 \\
\hline 58 & $\begin{array}{l}\text { V. radiata var } \\
\text { sublobata }\end{array}$ & 38 & 36.29 & 24.98 & 3.41 & 11.62 & 3.17 & 30.30 & 4.10 & 2.00 & 0.16 & 2.63 & 1.73 & 2.20 & 1.23 & 0.04 \\
\hline
\end{tabular}




\begin{tabular}{|c|c|c|c|c|c|c|c|c|c|c|c|c|c|c|c|c|}
\hline 59 & Pant Mung-5 & 38 & 45.29 & 15.38 & 2.61 & 9.82 & 3.97 & 23.70 & 7.53 & 5.00 & 0.21 & 4.52 & 3.51 & 4.84 & 1.40 & 0.20 \\
\hline 60 & Harsha & 40 & 48.69 & 26.18 & 3.01 & 5.42 & 3.77 & 18.10 & 6.50 & 3.95 & 0.20 & 3.93 & 2.77 & 5.00 & 1.60 & 0.09 \\
\hline 61 & KM-2241 & 44 & 54.89 & 28.38 & 2.61 & 12.02 & 4.16 & 40.10 & 6.64 & 4.19 & 0.19 & 4.02 & 2.98 & 3.35 & 1.32 & 0.15 \\
\hline 62 & OBGG-52 & 38 & 48.49 & 20.58 & 2.41 & 10.62 & 4.16 & 39.70 & 5.72 & 4.00 & 0.18 & 3.69 & 2.87 & 2.90 & 1.35 & 0.08 \\
\hline 63 & $12 / 333$ & 38 & 58.29 & 26.38 & 4.01 & 22.42 & 4.16 & 59.30 & 7.39 & 4.52 & 0.21 & 4.12 & 3.15 & 3.29 & 1.37 & 0.35 \\
\hline 64 & SML-668 & 34 & 61.09 & 26.78 & 3.81 & 18.62 & 3.17 & 45.10 & 9.52 & 5.44 & 0.18 & 5.07 & 3.33 & 4.86 & 1.52 & 0.67 \\
\hline 65 & 45-8-1 & 40 & 51.09 & 39.98 & 3.61 & 17.62 & 3.97 & 49.30 & 7.56 & 4.61 & 0.21 & 4.49 & 3.58 & 3.32 & 1.39 & 0.30 \\
\hline 66 & AKM-9904 & 38 & 57.49 & 34.18 & 3.81 & 16.62 & 3.57 & 45.30 & 7.16 & 4.27 & 0.18 & 4.22 & 3.28 & 3.35 & 1.31 & 0.21 \\
\hline 67 & Pre Dred & 38 & 49.09 & 21.18 & 3.81 & 12.42 & 3.97 & 56.50 & 7.48 & 4.60 & 0.18 & 4.55 & 3.14 & 3.29 & 1.44 & 0.36 \\
\hline 68 & Samrat & 47 & 50.09 & 29.18 & 2.81 & 8.22 & 3.97 & 31.50 & 6.67 & 4.29 & 0.18 & 3.98 & 3.29 & 4.24 & 1.51 & 0.19 \\
\hline 69 & NDM 5-3 & 42 & 64.89 & 41.78 & 4.61 & 24.42 & 3.77 & 62.90 & 7.72 & 4.09 & 0.18 & 4.22 & 3.17 & 3.59 & 1.40 & 0.40 \\
\hline 70 & МH-429 & 34 & 62.29 & 40.58 & 4.81 & 16.82 & 3.77 & 55.10 & 7.66 & 4.44 & 0.15 & 4.30 & 3.17 & 4.46 & 1.49 & 0.53 \\
\hline 71 & NIABM & 33 & 53.09 & 19.98 & 2.61 & 9.22 & 3.57 & 24.90 & 7.76 & 4.63 & 0.20 & 4.24 & 3.52 & 3.77 & 1.41 & 0.14 \\
\hline 72 & VC 1997 A & 38 & 60.69 & 33.98 & 3.61 & 17.02 & 3.77 & 49.10 & 9.31 & 6.01 & 0.22 & 5.54 & 4.02 & 5.85 & 1.72 & 0.68 \\
\hline 73 & VC $6040 \mathrm{~A}$ & 40 & 47.29 & 14.18 & 2.81 & 9.42 & 3.77 & 30.50 & 7.28 & 4.18 & 0.16 & 4.62 & 3.35 & 5.09 & 1.53 & 0.26 \\
\hline 74 & VC-7960 & 38 & 40.29 & 12.98 & 2.81 & 9.42 & 3.77 & 28.10 & 6.96 & 4.87 & 0.14 & 4.41 & 3.16 & 5.16 & 1.60 & 0.30 \\
\hline 75 & HUM-16 & 34 & 39.89 & 17.98 & 2.61 & 8.02 & 3.77 & 27.30 & 7.79 & 4.61 & 0.16 & 4.90 & 3.12 & 5.15 & 1.66 & 0.32 \\
\hline 76 & NDM-1 & 40 & 69.49 & 45.38 & 4.81 & 25.82 & 3.97 & 63.50 & 7.03 & 4.99 & 0.23 & 3.81 & 3.49 & 3.49 & 1.25 & 0.47 \\
\hline 77 & NDM 5-31 & 38 & 59.29 & 32.78 & 4.01 & 17.22 & 4.16 & 43.10 & 7.60 & 4.55 & 0.21 & 4.13 & 3.37 & 4.17 & 1.44 & 0.35 \\
\hline 78 & МH-418 & 42 & 56.09 & 32.98 & 4.01 & 18.62 & 3.97 & 61.30 & 6.56 & 4.54 & 0.21 & 3.79 & 3.15 & 3.00 & 1.17 & 0.22 \\
\hline 79 & ML-133 & 40 & 56.09 & 43.38 & 3.81 & 14.42 & 4.37 & 58.70 & 7.47 & 5.13 & 0.18 & 4.05 & 3.19 & 3.52 & 1.20 & 0.38 \\
\hline 80 & CN 9-5 & 31 & 58.09 & 33.98 & 3.01 & 18.22 & 4.37 & 61.10 & 8.31 & 4.95 & 0.17 & 4.48 & 3.57 & 5.11 & 1.34 & 0.51 \\
\hline 81 & Mung Local & 34 & 48.09 & 26.78 & 3.01 & 18.82 & 3.57 & 55.10 & 6.35 & 4.75 & 0.20 & 4.32 & 3.13 & 3.07 & 1.15 & 0.20 \\
\hline 82 & Pusa Ratna & 34 & 37.49 & 18.98 & 2.40 & 7.22 & 3.97 & 26.90 & 6.76 & 4.34 & 0.17 & 4.11 & 2.98 & 3.94 & 1.39 & 0.12 \\
\hline 83 & Pusa-9531 & 42 & 51.49 & 23.78 & 2.61 & 11.82 & 3.97 & 38.70 & 6.09 & 4.36 & 0.18 & 3.96 & 3.36 & 3.79 & 1.35 & 0.17 \\
\hline 84 & Pusa-672 & 38 & 52.29 & 41.58 & 3.61 & 13.42 & 3.37 & 35.90 & 7.51 & 4.65 & 0.20 & 4.47 & 3.22 & 2.89 & 1.19 & 0.12 \\
\hline 85 & IPM 02-19 & 36 & 50.37 & 29.96 & 3.79 & 17.23 & 4.07 & 57.54 & 7.34 & 4.47 & 0.17 & 4.09 & 3.15 & 3.44 & 1.31 & 0.33 \\
\hline 86 & IPM 02-17 & 39 & 55.17 & 30.16 & 3.59 & 20.83 & 3.87 & 59.34 & 7.28 & 4.65 & 0.17 & 4.10 & 2.98 & 3.70 & 1.35 & 0.40 \\
\hline 87 & MH-521 & 39 & 53.37 & 20.96 & 3.79 & 12.23 & 3.87 & 39.94 & 7.12 & 4.62 & 0.19 & 3.82 & 3.31 & 3.84 & 1.37 & 0.25 \\
\hline 88 & MH-318 & 41 & 53.97 & 25.76 & 3.99 & 14.43 & 4.07 & 49.14 & 7.16 & 4.17 & 0.17 & 3.74 & 3.06 & 3.50 & 1.36 & 0.25 \\
\hline 89 & TM 96-2 & 35 & 61.37 & 44.96 & 4.99 & 25.63 & 4.47 & 69.34 & 9.15 & 4.94 & 0.21 & 4.45 & 3.40 & 4.11 & 1.37 & 0.58 \\
\hline 90 & COGG-912 & 41 & 65.77 & 32.96 & 4.39 & 19.43 & 3.87 & 50.34 & 7.80 & 5.26 & 0.24 & 4.95 & 3.16 & 5.09 & 1.56 & 0.62 \\
\hline
\end{tabular}




\begin{tabular}{|c|c|c|c|c|c|c|c|c|c|c|c|c|c|c|c|c|}
\hline 91 & RMG-991 & 39 & 51.57 & 29.96 & 3.59 & 15.63 & 3.67 & 44.74 & 5.97 & 3.88 & 0.14 & 3.95 & 2.81 & 3.05 & 1.20 & 0.09 \\
\hline 92 & Pusa-871 & 39 & 47.37 & 17.56 & 3.19 & 12.03 & 3.47 & 34.34 & 6.54 & 4.18 & 0.16 & 3.79 & 3.17 & 3.51 & 1.40 & 0.13 \\
\hline 93 & PM 06-32 & 39 & 81.37 & 61.36 & 4.39 & 38.03 & 3.87 & 65.94 & 6.33 & 4.36 & 0.19 & 3.66 & 3.13 & 2.87 & 1.15 & 0.49 \\
\hline 94 & PM 06-33 & 41 & 62.77 & 35.96 & 3.79 & 20.03 & 4.07 & 49.74 & 7.39 & 4.63 & 0.21 & 3.98 & 3.30 & 4.13 & 1.44 & 0.41 \\
\hline 95 & PM 06-34 & 48 & 69.37 & 41.36 & 4.39 & 24.63 & 3.67 & 62.94 & 7.72 & 4.18 & 0.17 & 4.10 & 3.29 & 3.67 & 1.34 & 0.40 \\
\hline 96 & PM 06-35 & 44 & 59.97 & 25.16 & 3.19 & 14.43 & 3.67 & 42.54 & 7.21 & 4.20 & 0.19 & 3.69 & 3.17 & 3.59 & 1.35 & 0.21 \\
\hline 97 & PM 06-36 & 39 & 67.17 & 46.36 & 3.79 & 17.23 & 4.87 & 58.34 & 7.59 & 4.53 & 0.21 & 3.77 & 3.25 & 3.60 & 1.39 & 0.40 \\
\hline 98 & PM 06-37 & 41 & 72.77 & 47.36 & 5.79 & 29.83 & 3.67 & 63.34 & 6.75 & 4.78 & 0.22 & 3.70 & 3.35 & 3.40 & 1.34 & 0.47 \\
\hline 99 & PM 06-39 & 39 & 53.77 & 37.16 & 4.19 & 19.83 & 3.47 & 39.94 & 6.64 & 4.33 & 0.20 & 4.06 & 3.34 & 3.30 & 1.34 & 0.16 \\
\hline 100 & PM 06-43 & 41 & 64.77 & 34.16 & 5.39 & 3.83 & 5.07 & 57.14 & 6.85 & 4.26 & 0.19 & 3.68 & 3.09 & 3.23 & 1.35 & 0.27 \\
\hline 101 & PM 06-46 & 41 & 81.17 & 43.76 & 5.39 & 30.23 & 5.67 & 88.14 & 6.83 & 4.66 & 0.19 & 4.02 & 3.19 & 3.71 & 1.36 & 0.71 \\
\hline 102 & КM 09-174 & 41 & 54.57 & 34.36 & 4.39 & 18.63 & 3.27 & 47.14 & 8.81 & 4.80 & 0.18 & 4.47 & 3.47 & 4.36 & 1.34 & 0.48 \\
\hline 103 & KM 09-182 & 42 & 55.17 & 28.76 & 3.79 & 18.63 & 3.87 & 41.34 & 7.43 & 4.54 & 0.18 & 4.29 & 3.25 & 3.16 & 1.22 & 0.17 \\
\hline 104 & SM 10-505 & 39 & 48.97 & 21.96 & 3.39 & 13.03 & 3.67 & 33.94 & 7.15 & 4.46 & 0.16 & 3.89 & 3.22 & 3.23 & 1.28 & 0.13 \\
\hline 105 & SM 10-503 & 41 & 49.97 & 21.56 & 3.99 & 15.03 & 4.47 & 34.14 & 7.23 & 4.38 & 0.18 & 3.76 & 3.04 & 3.40 & 1.29 & 0.14 \\
\hline 106 & SM 10-533 & 41 & 49.57 & 18.96 & 3.59 & 23.63 & 3.27 & 34.34 & 9.15 & 4.80 & 0.20 & 4.56 & 3.49 & 4.34 & 1.48 & 0.39 \\
\hline 107 & SM 10-529 & 39 & 57.17 & 41.76 & 4.59 & 24.43 & 3.47 & 53.74 & 9.26 & 4.77 & 0.22 & 4.21 & 3.32 & 4.98 & 1.42 & 0.54 \\
\hline 108 & V. trilobata $\mathrm{X}$ & 41 & 62.77 & 37.76 & 5.19 & 25.83 & 4.27 & 57.54 & 7.35 & 4.73 & 0.19 & 3.98 & 3.24 & 3.49 & 1.30 & 0.36 \\
\hline 109 & V. sublobataX & 42 & 56.37 & 29.36 & 5.59 & 20.83 & 3.87 & 51.34 & 7.36 & 4.73 & 0.19 & 3.97 & 3.26 & 2.56 & 1.32 & 0.20 \\
\hline 110 & V. silvestris $\mathbf{X}$ & 42 & 57.77 & 42.16 & 4.79 & 20.83 & 3.47 & 49.34 & 5.10 & 4.44 & 0.23 & 4.19 & 2.76 & 3.81 & 1.50 & 0.22 \\
\hline 111 & IPM 02-19 & 47 & 34.17 & 25.16 & 3.59 & 19.83 & 3.27 & 40.54 & 6.50 & 4.39 & 0.18 & 4.17 & 3.26 & 3.17 & 1.29 & 0.14 \\
\hline 112 & PM 06-50 & 36 & 36.97 & 27.76 & 3.19 & 11.43 & 3.27 & 29.94 & 7.39 & 4.29 & 0.18 & 4.11 & 3.11 & 4.07 & 1.53 & 0.20 \\
\hline \multicolumn{2}{|c|}{ Mean } & 38.38 & 58.71 & 29.86 & 4.10 & 15.76 & 3.96 & 44.15 & 7.59 & 4.66 & 0.20 & 4.24 & 3.29 & 3.85 & 1.36 & 0.31 \\
\hline \multirow[t]{2}{*}{ Range } & Min. & 31 & 34.17 & 11.18 & 2.35 & 3.83 & 3.17 & 18.10 & 4.10 & 2.00 & 0.14 & 2.63 & 1.73 & 2.20 & 1.12 & 0.04 \\
\hline & Max. & 48 & 84.49 & 61.36 & 9.25 & 38.03 & 5.67 & 88.14 & 10.32 & 6.01 & 0.28 & 5.55 & 4.02 & 5.85 & 1.72 & 0.71 \\
\hline
\end{tabular}


Table.3 List of superior genotypes for different characters

\begin{tabular}{|c|c|c|c|c|}
\hline S. No. & Character & Rank & Genotypes & Values \\
\hline \multirow[t]{5}{*}{1.} & \multirow[t]{5}{*}{ Days to $50 \%$ flowering } & 1. & UPM 98-1 & 31.00 \\
\hline & & 2. & CN 9-5 & 31.00 \\
\hline & & 3. & VC $6769(57-99)$ & 32.00 \\
\hline & & 4. & Mauritius Local & 32.00 \\
\hline & & 5. & Pusa Vishal, Mauritius Mung-1 & 32.00 \\
\hline \multirow[t]{5}{*}{2.} & \multirow[t]{5}{*}{ Plant height $(\mathrm{cm})$} & 1. & IPM 02-19 & 34.17 \\
\hline & & 2. & Vigna radiata var sublobata & 36.29 \\
\hline & & 3. & PM 06-50 & 36.97 \\
\hline & & 4. & Pusa ratna & 37.49 \\
\hline & & 5. & HUM-16 & 39.89 \\
\hline \multirow[t]{5}{*}{3.} & \multirow{5}{*}{ Number of leaves } & 1. & NH 54 & 11.18 \\
\hline & & 2. & VC 7960 & 12.98 \\
\hline & & 3. & VC $6040 \mathrm{~A}$ & 14.18 \\
\hline & & 4. & Pant mung-5 & 15.38 \\
\hline & & 5. & PM 06-45 & 16.70 \\
\hline \multirow[t]{5}{*}{4.} & \multirow[t]{5}{*}{ Number of branches } & 1. & VC 7960-88 & 2.35 \\
\hline & & 2. & Pusa ratna & 2.40 \\
\hline & & 3. & OBGG-52 & 2.41 \\
\hline & & 4. & PM 08-2 & 2.54 \\
\hline & & 5. & NM 1 (Mutant) & 2.55 \\
\hline \multirow[t]{5}{*}{5.} & \multirow[t]{5}{*}{ Number of clusters } & 1. & PM 06-32 & 38.03 \\
\hline & & 2. & PM 06-46 & 30.23 \\
\hline & & 3. & PM 06-37 & 29.83 \\
\hline & & 4. & Barimung-4 & 28.59 \\
\hline & & 5. & BDYR-1 & 26.19 \\
\hline \multirow[t]{5}{*}{6.} & \multirow[t]{5}{*}{ Number of pods per cluster } & 1. & PM 06-46 & 5.67 \\
\hline & & 2. & PM 06-51 & 5.39 \\
\hline & & 3. & PM 06-43 & 5.07 \\
\hline & & 4. & PM 06-36 & 4.87 \\
\hline & & 5. & PM-03-2, PM 03-16, PM 06-48 & 4.59 \\
\hline \multirow[t]{5}{*}{7.} & \multirow[t]{5}{*}{ Total number of pods per plant } & 1. & PM 06-46 & 88.14 \\
\hline & & 2. & TM 96-2 & 69.34 \\
\hline & & 3. & PM 06-32 & 65.94 \\
\hline & & 4. & NDM-1 & 63.50 \\
\hline & & 5. & PM 06-37 & 63.34 \\
\hline 8. & Pod length $(\mathrm{cm})$ & 1. & NH-54 & 10.32 \\
\hline
\end{tabular}


Int.J.Curr.Microbiol.App.Sci (2020) 9(1): 780-790

\begin{tabular}{|c|c|c|c|c|}
\hline & & 2. & SML-668 & 9.52 \\
\hline & & 3. & МH-318 & 9.46 \\
\hline & & 4. & UPM 02-16 & 9.42 \\
\hline & & 5. & VC 1997 A & 9.31 \\
\hline \multirow[t]{5}{*}{9.} & \multirow{5}{*}{ Pod diameter (mm) } & 1. & VC $1997 \mathrm{~A}$ & 6.01 \\
\hline & & 2. & Barimung-5 & 5.90 \\
\hline & & 3. & VC $6790 \mathrm{~A}$ & 5.71 \\
\hline & & 4. & SML-668 & 5.44 \\
\hline & & 5. & PM 03-20 & 5.40 \\
\hline \multirow[t]{5}{*}{10.} & \multirow[t]{5}{*}{ Pod wall thickness (mm) } & 1. & RMG 991 & 0.14 \\
\hline & & 2. & VC 7960 & 0.14 \\
\hline & & 3. & MH 429 & 0.15 \\
\hline & & 4. & PM 08-1 & 0.16 \\
\hline & & 5. & Vigna radiata var sublobata & 0.16 \\
\hline \multirow[t]{5}{*}{11.} & \multirow[t]{5}{*}{ Seed length (mm) } & 1. & VC $6790 \mathrm{~A}$ & 5.55 \\
\hline & & 2. & VC 1997 A & 5.54 \\
\hline & & 3. & PM 03-23 & 5.18 \\
\hline & & 4. & SML-668 & 5.07 \\
\hline & & 5. & NH-54 & 5.03 \\
\hline \multirow[t]{5}{*}{12.} & \multirow[t]{5}{*}{ Seed diameter $(\mathrm{mm})$} & 1. & VC 1997 A & 4.02 \\
\hline & & 2. & UPM-98 & 3.82 \\
\hline & & 3. & VC $6790 \mathrm{~A}$ & 3.81 \\
\hline & & 4. & Barimung-5 & 3.81 \\
\hline & & 5. & MH-318 & 3.73 \\
\hline \multirow[t]{5}{*}{13.} & \multirow[t]{5}{*}{100 -seed weight (g) } & 1. & VC 1997 A & 5.85 \\
\hline & & 2. & Barimung-5 & 5.52 \\
\hline & & 3. & VC $6790 \mathrm{~A}$ & 5.31 \\
\hline & & 4. & MH-318 & 5.24 \\
\hline & & 5. & VC-7960 & 5.16 \\
\hline \multirow[t]{5}{*}{14.} & \multirow[t]{5}{*}{ Seed density (g/ml) } & 1. & VC 1997 A & 1.72 \\
\hline & & 2. & HUM-16 & 1.66 \\
\hline & & 3. & VC-7960, Harsha & 1.60 \\
\hline & & 4. & COGG-912, MH-318 & 1.56 \\
\hline & & 5. & Barisal local & 1.54 \\
\hline \multirow[t]{5}{*}{15.} & \multirow[t]{5}{*}{ Seed yield per plot (kg) } & 1. & PM 06-46 & 0.71 \\
\hline & & 2. & MH-318 & 0.69 \\
\hline & & 3. & VC 1997 A & 0.68 \\
\hline & & 4. & SML-668 & 0.67 \\
\hline & & 5. & COGG-912 & 0.62 \\
\hline
\end{tabular}


The efficiency of a breeding programme for the improvement of quantitative traits depends to a large extent on magnitude of variability present in the available germplasm (Hanna et al., 1999). Goodman (1999) emphasized the importance of breeding stock and germplasm accessions by suggesting that although lines may be unpromising phenotypically but may contain untapped alleles or allelic combinations that could be utilized for plant breeding with adequate investment in conventional and marker assisted selection. The list of promising genotypes in respect of various characters has been presented in table 3 , which may be used for parental selection in breeding programmes for improvement of yield and to improve various qualitative and quantitative characteristics in mungbean.

\section{Acknowledgements}

The author acknowledges the support and valuable guide from the Advisor, Head of department of Genetics and Plant Breeding, Dean College of Agriculture and Director of Research, G.B. Pant University of Agriculture and Technology, Pantnagar for their valuable suggestions and providing time being facilities for conducting the experiment.

\section{References}

Goodman, M.M. 1999. Broadening the genetic diversity in maize breeding by use of exotic germplasm. In: National Symposium on Onion-Garlic Production and Post Harvest management, Challenges and Strategies. Nashik. pp. 38-40.

Federer, W.T. 1961 Augmented designs with one way elimination of heterogeneity. Biometrics. International Biometric Society. 17(3):447-473.

Hanna, W., Ozias- Akins, P. and Roche, D. 1999. Apomixes and Heterosis. In: J. K. Coors and S.Pandey(eds.), Genetics and Exploitation of Heterosis in crops. American Society of Agronomy, Inc. Wisconsin. U.S.A. pp.335-342.

Lakhanpaul, S.S.C. and Bhat, K.V. (2000) Random amplified polymorphic DNA (RAPD) analysis in Indian mungbean (Vigna radiata (L.) Wilczek) cultivars. Genetica. 109, 227-234.

Paroda, R.S. and Arora, R.K. 1991. Plant genetic resources: general perspective. In Plant genetic resources; conservation and management. New Delhi, Malhotra publishing house. pp.1-23.

Rao, V.S., Leela, R., Swaminathan, M. and Parpia, H.A.B. 1964. The nutritive value of the proteins of leguminous seed. Indian J. Nutr. Diet., 1: 304.

Singh DP and Ahlawat IPS. 2005. Greengram (Vigna radiata) and blackgram ( $V$. mungo) improvement in India: past, present and future prospects. Indian $J$ Agricultural Sciences 75(5): 243-250.

\section{How to cite this article:}

Sarfraz Ahmad and Vikas Belwal. 2020. Morphological Characterization and Evaluation of Mungbean [Vigna radiate (L.) Wilczek] Germplasm for Various Yield Attributing Traits. Int.J.Curr.Microbiol.App.Sci. 9(01): 780-790. doi: https://doi.org/10.20546/ijcmas.2020.901.084 